\title{
Energie- und Klimapolitik im Vertrag von Lissabon: Legitimationserweiterung für wachsende Herausforderungen
}

\author{
Severin Fischer*
}

Die Entwicklung einer erfolgreichen Energiepolitik, die Umstrukturierung der Energiemärkte und die Wahrnehmung einer Vorbildfunktion im Klimaschutz werden immer häufiger als neue Leitmotive eines zukunftsfähigen Europas aufgeführt. Stellte bis heute die Errichtung eines Gemeinsamen Marktes die vielleicht wichtigste Triebfeder des europäischen Integrationsprozesses dar, so erhält derzeit die Formulierung einer gemeinschaftlichen Energie- und Klimapolitik einen ähnlichen Stellenwert. Sie stand im Mittelpunkt der Beschlüsse des Europäischen Rates vom März 2007, ${ }^{1}$ avancierte zur Priorität der französischen Ratspräsidentschaft im zweiten Halbjahr $2008^{2}$ und wird ebenfalls zentrales Handlungsfeld der Anfang 2009 antretenden tschechischen Ratspräsidentschaft sein. Im Lichte all dieser erfolgversprechenden Entwicklungen bleibt immer häufiger unberücksichtigt, dass die Europäische Union bislang nur über ein sehr schwach ausgeprägtes primärrechtliches Portfolio in der Energiepolitik verfügt. Dies ist jedoch nicht unbedeutend, regeln doch zwei entscheidende Grundsätze die Kompetenzverteilung zwischen der Union und ihren Mitgliedstaaten und damit die Legitimation zur Entwicklung von Sekundärrecht: das Prinzip der , begrenzten Einzelermächtigung' und das Prinzip der ,Subsidiarität ‘3 Anders als die souveränen Nationalstaaten, benötigt die Europäische Union eine ausdrückliche Rechtsgrundlage in den Verträgen, um gesetzgeberisch tätig werden zu können. ${ }^{4}$ Ein solches explizites primärrechtliches Fundament für den Bereich Energie- und Klimapolitik wurde erstmals durch den Vertrag über eine Verfassung für Europa $(\mathrm{VVE})^{5}$ geschaffen und mit weiteren Ergänzungen in den Vertrag von Lissabon integriert. Sollte der Vertrag den schwierigen Parcours der Ratifikation in den 27 Mitgliedstaaten überstehen, dürfte die Europäische Union erstmals im Besitz einer energiepolitischen Kompetenz sein.

Diese Feststellung erscheint aus zwei Gründen jedoch nicht vollkommen richtig: Einerseits würde diese Auffassung implizieren, dass Europa in der Energiepolitik bislang handlungsunfähig war. Andererseits würde es bedeuten, dass die Europäische Union im Falle einer Ablehnung des Vertrags von Lissabon wieder auf den Zustand eines energiepolitischen ,Habenichts` zurückgeworfen würde. Da die europäische Staatengemeinschaft jedoch bereits in

* Severin Fischer, Wissenschaftlicher Mitarbeiter, Institut für Europäische Politik, Berlin. Dieser Aufsatz ist im Rahmen eines gemeinsamen Projekts mit der ASKO EUROPA-STIFTUNG entstanden.

1 Vgl. Rat der Europäischen Union: Europäischer Rat (Brüssel), 8./9. März 2007. Schlussfolgerungen des Vorsitzes, Dok. 7224/1/07 REV 1.

2 Vgl. Französische Ratspräsidentschaft: Arbeitsprogramm 1. Juli-31. Dezember 2008. Ein Europa, das handelt, um den aktuellen Herausforderungen gerecht zu werden, abrufbar unter: http://www.ue2008.fr/webdav/site/ PFUE/shared/ProgrammePFUE/Programme_DE.pdf (letzter Zugriff: 25.11.2008). Ausführlicher Institut für Europäische Politik (Hrsg.): EU-27 Watch, No. 7, Berlin 2008.

3 Beide Prinzipien wurden durch den Vertrag von Maastricht als Grundlagen für eine Handlungsermächtigung festgelegt und finden sich in Art. 5 Abs. 1 und Art. 5 Abs. 2 des Vertrags zur Gründung der Europäischen Gemeinschaft in der Fassung des Vertrages von Nizza (EGV), in: Amtsblatt der Europäischen Union, Nr. C 321 vom 29. Dezember 2006. Siehe darüber hinaus unter anderem Thomas Oppermann: Europarecht, 2. Auflage, München 1999, S. 197-204; Claus Dieter Classen: Einführung, in: Deutscher Taschenbuch Verlag (Hrsg.): Europa-Recht, 19. Auflage, München 2004, S. XI-XXVI, hier S. XVI-XVII.

4 Vgl. Gert Nicolaysen: Europarecht I. Die Europäische Integrationsverfassung, 2. Auflage, Baden-Baden 2002, S. $270-271$.

5 Der VVE ist veröffentlicht in: Amtsblatt der Europäischen Union, Nr. C 310 vom 16. Dezember 2004. 
der Vergangenheit zahlreiche - mit mehr oder weniger Erfolg beschiedene - energiepolitische Regelungen erlassen hat und dies derzeit in umfangreicher und weit ehrgeizigerer Weise fortsetzt, ${ }^{6}$ besteht eine Diskrepanz zwischen Primärrecht und Wirklichkeit, die einer tiefer gehenden Betrachtung bedarf. Aus diesem Grund erscheint es ratsam, einen Blick auf die primärrechtliche Situation einer sich ständig weiterentwickelnden EU-Energie- und Klimapolitik zu werfen und die Neuerungen des Vertrags von Lissabon unter diesem Gesichtspunkt genauer zu untersuchen. Dabei sind nicht nur die primärrechtlichen Änderungen als solche wichtig, gleichermaßen muss auch ein Blick auf die daraus resultierenden legitimationsbildenden und psychologischen Effekte geworfen werden, die für die zukünftige Entwicklung eines Politikfelds von entscheidender Bedeutung sind. Gerade im Prozess der Verhandlung umfangreicher Gesetzgebungspakete und der Aushandlung internationaler Vereinbarungen im Rahmen der Energiepolitik spielen diese Faktoren eine nicht zu unterschätzende Rolle. Gleiches gilt für die komplizierten Verhandlungen eines Post-Kyoto-Abkommens zum Klimaschutz, das bis zum Gipfel 2009 in Kopenhagen entwickelt werden soll. Schließlich bedarf es, auch aufgrund der Unsicherheit über die endgültige Ratifikation des Vertrags von Lissabon, eines Blicks auf Handlungsoptionen, die außerhalb einer Vertragsrevision liegen. In diesem Kontext ist abschließend der Frage nachzugehen, ob die Europäische Union den wachsenden Herausforderungen des Klimawandels, der Ressourcenverknappung und der zunehmenden Einbindung von Energiepolitik in die internationalen Beziehungen gerecht werden kann, ohne eine ausdrückliche primärrechtliche Kompetenz für diesen Aufgabenbereich erhalten zu haben.

\section{Die primärrechtlichen Änderungen zur Energie- und Klimapolitik im Vertrag von Lissabon}

Auch wenn der europäische Integrationsprozess seinen Ursprung in der Bearbeitung energiepolitischer Fragen im Rahmen der Montanunion und des EURATOM-Vertrags hatte, besitzt die Europäische Union bis zum heutigen Tage keine explizite Kompetenz zur Durchsetzung einer europäischen Energiepolitik. ${ }^{7}$ Konkrete Maßnahmen, etwa zur Bewältigung der Ölkrisen in den 1970er Jahren, bedurften der Einstimmigkeit der Mitgliedstaaten im Rat und hatten in ihrer Folgewirkung meist den Charakter eines Placebos. ${ }^{8}$ Nichtsdestotrotz wäre es verkürzt zu behaupten, die Europäische Union habe kein energiepolitisches Profil. Im Rahmen der Kompetenzerweiterung um den Bereich Umwelt in den 1980er Jahren und durch das handlungsleitende Integrationsprojekt der Entwicklung eines europäischen Binnenmarktes für alle Produkte, so auch Strom, Erdöl und Erdgas, hatte sich die Europäische Union ihre Ermächtigung für die Gestaltung energiepolitischer Maßnahmen gewissermaßen selbst geschaffen. Darüber hinaus war die Regulierung des Ausbaus grenzüberschreitender Infrastruktur im Rahmen des Kapitels „Transeuropäische Netze“ ein Kernthema des späteren Integrationsprozesses, dem sich die Kommission auch im Energiesektor annahm.

Mit dem VVE wurde dem Bereich „Energie“ erstmals ein eigener Abschnitt gewidmet, welcher nun beinahe unverändert in den Besitzstand des Vertrags von Lissabon übernommen wurde. ${ }^{9}$ So wird in Titel XXI, bestehend aus einem einzigen Artikel, ${ }^{10}$ zunächst die

6 Siehe hierzu ausführlicher: Oliver Geden: Die Energie- und Klimapolitik der EU - zwischen Implementierung und strategischer Neuorientierung, in: integration 4/2008, S. 353-364.

7 Vgl. Oliver Geden/Severin Fischer: Die Energie- und Klimapolitik der Europäischen Union. Bestandsaufnahme und Perspektiven, Baden-Baden 2008.

8 Siehe hierzu ausführlicher: Jürgen Grunwald: Das Energierecht der Europäischen Gemeinschaften. EGKSEURATOM-EG. Grundlagen - Geschichte - Geltende Regelungen, Berlin 2003, vor allem S. 47ff.

9 Art. III-256 VVE.

10 Art. 194 des Vertrags über die Arbeitsweise der Europäischen Union (AEUV) in der konsolidierten Fassung des Lissabonner Vertrages, in: Amtsblatt der Europäischen Union, Nr. C 115 vom 9. Mai 2008. 
grundsätzliche Ausrichtung einer europäischen Energiepolitik bestimmt. Diese verfolge „im Geiste der Solidarität zwischen den Mitgliedstaaten" die Verwirklichung und das Funktionieren des Binnenmarktes unter Berücksichtigung der Erfordernisse der Umweltverträglichkeit. ${ }^{11}$ Dies entspricht der historischen Grundlage europäischer Energiepolitik, die sich bislang in erster Linie auf Maßnahmen zur Angleichung der Rechtsvorschriften im Binnenmarkt $^{12}$ und der Umweltpolitik ${ }^{13}$ berufen musste. Die explizite Nennung des Solidaritätsgedankens erfolgte auf Druck der polnischen Delegation bei den Beratungen zum neuen Vertragswerk. Dabei spielten die Erfahrungen aus dem ukrainisch-russischen Gasstreit im Frühjahr 2006 eine wichtige Rolle. ${ }^{14}$ Die Nennung des Solidaritätsgedankens wiederholt sich in Art. 122 Abs. 1 AEUV, wobei hier explizit gemeinschaftliche Maßnahmen bei Versorgungsengpässen ,im Energiebereich“ thematisiert werden.

Als Zielsetzungen für eine zukünftige europäische Energiepolitik formulierten die Verfasser des Vertrags vier Bereiche: die „Sicherstellung des Funktionierens des Energiemarktes“, die „Gewährleistung der Energieversorgungssicherheit in der Union“, die Förderung von Energieeffizienz, von Energieeinsparungen und die Entwicklung neuer und erneuerbarer Energiequellen sowie die „Förderung der Interkonnektion der Energienetze“ . ${ }^{15}$ Der Netzausbau als Zielsetzung der Union in der Energiepolitik stellt damit eine Ergänzung im Verhältnis zum Energieabschnitt des VVE dar. ${ }^{16}$ Für das gesamte Politikfeld sieht der Vertrag die Anwendung des ordentlichen Gesetzgebungsverfahrens vor. Eine Ausnahme stellt das Recht jedes einzelnen Mitgliedstaates dar, ,die Bedingungen für die Nutzung seiner Energieressourcen, seine Wahl zwischen verschiedenen Energiequellen und die allgemeine Struktur seiner Energieversorgung zu bestimmen". ${ }^{17}$ Maßnahmen, die einen überwiegend steuerlichen Charakter besitzen, müssen zudem vom Rat einstimmig angenommen werden.

Schließlich finden sich auch außerhalb des Energietitels Änderungen, die eine neue Ausrichtung europäischer Energie- und Klimapolitik ermöglichen. Innerhalb des Umwelttitels wird erstmals das Ziel formuliert, die Bekämpfung des Klimawandels zu einem Teil der gemeinschaftlichen Umweltpolitik zu machen. ${ }^{18}$ Gleichzeitig wird im Umwelttitel jedoch die Souveränität der Mitgliedstaaten über Struktur und Wahl der Energieträger beibehalten ${ }^{19}$ und findet sich somit zweimal im Vertrag wieder: sowohl im Energie- als auch im Umwelttitel.

Die Neuerungen im Vertrag von Lissabon zur Entwicklung einer integrierten Energieund Klimapolitik stellen eine substanzielle kompetenzrechtliche Neuausstattung der Gemeinschaft in diesem Politikfeld dar. In allen drei Bereichen des ,energiepolitischen Zieldreiecks“ von Umweltverträglichkeit, Wettbewerbsfähigkeit und Versorgungssicherheit finden sich deutliche Anpassungen an die tatsächliche Handlungsbreite und die globalen Gestaltungserfordernisse. ${ }^{20}$ Dabei wird insbesondere der Bereich Versorgungssicherheit gestärkt,

11 Ebenda.

12 In den meisten Fällen durch Anwendung von Art. 95 EGV.

13 In den meisten Fällen durch Anwendung von Art. 175 EGV.

14 Vgl. Marc-Oliver Pahl: Umwelt, Energie und Landwirtschaft, in: Ingolf Pernice (Hrsg.): Der Vertrag von Lissabon: Reform der EU ohne Verfassung? Kolloquium zum 10. Geburtstag des WHI, Baden-Baden 2008, S. 205-209; Josefine Kuhlmann: Kompetenzrechtliche Neuerungen im europäischen Energierecht nach dem Vertrag von Lissabon, Working Paper des Europainstituts der Wirtschaftsuniversität Wien 79/2008, S. 22.

15 Art. 194 Abs. 1 AEUV.

16 Vgl. wiederum Art. III-256 VVE.

17 Art. 194 Abs. 2 AEUV.

18 Art. 191 Abs. 1 AEUV.

19 Art. 192 Abs. 2 Lit. c AEUV.

20 Mit dem ,energiepolitischen Zieldreieck“ wird die strategische Ausrichtung der Trias Umweltverträglichkeit, Wettbewerbsfähigkeit und Versorgungssicherheit unter teils wechselnden Begrifflichkeiten verstanden. Diese dominiert die Diskussion über moderne Energiepolitik sowohl auf nationaler als auch auf europäischer Ebene. Der Europäische Rat hat sie in seinen Schlussfolgerungen vom März 2007 als strategische Zielpunkte für sich definiert. Siehe dazu ausführlicher Geden/Fischer: Die Energie- und Klimapolitik der Europäischen Union, 2008, S. 13-22. 


\begin{tabular}{|c|c|c|c|}
\hline $\begin{array}{l}\text { Vertragsbasis } \\
\text { EGV } \\
\text { (Nizza) }\end{array}$ & Regelungsgegenstand & Neuerung & $\begin{array}{c}\text { Vertragsbasis } \\
\text { AEUV } \\
\text { (Lissabon) }\end{array}$ \\
\hline & \multicolumn{2}{|c|}{$\begin{array}{c}\text { Erster Teil, Titel I - Arten und Bereiche der Zuständigkeit } \\
\text { der Union }\end{array}$} & \\
\hline- & $\begin{array}{l}\text { Ausschließliche Zuständigkeit } \\
\text { der Union für die „Festlegung } \\
\text { der für das Funktionieren des } \\
\text { Binnenmarkts erforderlichen } \\
\text { Wettbewerbsregeln“ }\end{array}$ & neu eingeführt & $\begin{array}{l}\text { Art. } 3 \text { Abs. } 1 \\
\text { Lit. } b\end{array}$ \\
\hline \multirow[t]{2}{*}{ Art. 3 Lit. $1, \mathrm{o}, \mathrm{u}$} & $\begin{array}{l}\text { Geteilte Zuständigkeit für Um- } \\
\text { welt, Transeuropäische Netze } \\
\text { und Energie }\end{array}$ & $\begin{array}{l}\text { Bisher „Maßnahmen im Be- } \\
\text { reich Energie“ jetzt „Energie“ }\end{array}$ & Art. 4 Lit. e, h, i \\
\hline & \multicolumn{2}{|c|}{$\begin{array}{c}\text { Dritter Teil, Titel VIII - Die Wirtschafts- und } \\
\text { Währungspolitik }\end{array}$} & \\
\hline \multirow[t]{2}{*}{ Art. 100 Abs. 1} & $\begin{array}{l}\text { Außergewöhnliche Maßnah- } \\
\text { men bei Versorgungsschwierig- } \\
\text { keiten mit bestimmten Waren }\end{array}$ & $\begin{array}{l}\text { Explizite Nennung des Energie- } \\
\text { bereichs, Handlungen im } \\
\text { „Geiste der Solidarität“ }\end{array}$ & Art. 122 Abs. 1 \\
\hline & \multicolumn{2}{|c|}{ Dritter Teil, Titel XVI - Transeuropäische Netze } & \\
\hline \multirow[t]{2}{*}{ Art. $154-156$} & $\begin{array}{l}\text { Bestimmungen zur Förderung } \\
\text { und Weiterentwicklung trans- } \\
\text { europäischer Infrastruktur }\end{array}$ & & Art. $170-172$ \\
\hline & \multicolumn{2}{|c|}{ Dritter Teil, Titel XX - Umwelt } & \\
\hline \multirow[t]{2}{*}{ Art. 174} & Umweltpolitische Ziele & Bekämpfung des Klimawandels & Art. 191 \\
\hline & \multicolumn{2}{|c|}{ Dritter Teil, Titel XXI - Energie } & \\
\hline- & $\begin{array}{l}\text { Energiepolitik der Europä- } \\
\text { ischen Union }\end{array}$ & $\begin{array}{l}\text { Ziele: Sicherstellung des Funk- } \\
\text { tionierens des Energiemarkts; } \\
\text { Gewährleistung der Energie- } \\
\text { versorgungssicherheit; Förde- } \\
\text { rung der Energieeffizienz und } \\
\text { von Energieeinsparungen sowie } \\
\text { Entwicklung neuer und erneu- } \\
\text { erbarer Energiequellen; Förde- } \\
\text { rung der Interkonnektion der } \\
\text { Energienetze } \\
\text { Einschränkung: Mitgliedstaat- } \\
\text { liche Kontrolle über die Bedin- } \\
\text { gungen für die Nutzung der na- } \\
\text { tionalen Energieressourcen, die } \\
\text { Wahl zwischen Energiequellen } \\
\text { und die allgemeine Struktur sei- } \\
\text { ner Energieversorgung }\end{array}$ & Art. 194 \\
\hline
\end{tabular}




\begin{tabular}{|c|c|c|c|}
\hline $\begin{array}{c}\text { Vertragsbasis } \\
\text { EGV } \\
\text { (Nizza) }\end{array}$ & Regelungsgegenstand & Neuerung & $\begin{array}{c}\text { Vertragsbasis } \\
\text { AEUV } \\
\text { (Lissabon) }\end{array}$ \\
\hline & \multicolumn{2}{|c|}{ Protokolle } & \\
\hline- & $\begin{array}{l}\text { Protokoll über die Einfuhr in } \\
\text { den Niederländischen Antillen } \\
\text { raffinierter Erdölerzeugnisse in } \\
\text { die Europäische Union }\end{array}$ & $\begin{array}{l}\text { Neu hinzugefügt; Art. } 6 \text { Abs. } 1 \\
\text { führt an, dass Bestimmungen } \\
\text { revidiert werden können, wenn } \\
\text { die Europäische Union eine } \\
\text { Energiepolitik aufstellt. }\end{array}$ & Nr. 31 \\
\hline \multirow[t]{2}{*}{-} & $\begin{array}{l}\text { Protokoll über die finanziellen } \\
\text { Folgen des Ablaufs des EGKS- } \\
\text { Vertrags und über den For- } \\
\text { schungsfonds für Kohle und } \\
\text { Stahl }\end{array}$ & $\begin{array}{l}\text { Neu hinzugefügt; Übergang des } \\
\text { EGKS-Vermögens in den For- } \\
\text { schungsfonds für Kohle und } \\
\text { Stahl }\end{array}$ & Nr. 37 \\
\hline & \multicolumn{2}{|c|}{ Erklärungen } & \\
\hline- & $\begin{array}{l}\text { Erklärung zu Artikel } 194 \text { des } \\
\text { Vertrags über die Arbeitsweise } \\
\text { der Europäischen Union }\end{array}$ & $\begin{array}{l}\text { neu hinzugefügt; Art. } 194 \text { lässt } \\
\text { das Recht der Mitgliedstaaten } \\
\text { unberührt, nationale Maßnah- } \\
\text { men zur Sicherung ihrer Ener- } \\
\text { gieversorgung zu treffen. }\end{array}$ & 35. Erklärung \\
\hline
\end{tabular}

doch auch Umweltverträglichkeit und Wettbewerbsfähigkeit erfahren eine leichte Aufwertung. Für die Gewährleistung des Funktionierens des Wettbewerbsprinzips erhält die Europäische Union über Art. 3 Abs. 1 AEUV erstmals eine kodifizierte ausschließliche Zuständigkeit. Diese wurde zwar nicht explizit für die Strukturierung der Energiemärkte geschaffen, die Wirkung dieser Bestimmung dürfte aber bis in den Energiesektor hineinreichen. $\mathrm{Zu}$ beachten ist zudem, dass sich diese ausschließliche Kompetenz nur auf die Wettbewerbsregelungen und nicht auf den Binnenmarkt als solchen bezieht. Dies hat zur Folge, dass die Europäische Union zwar alleine für das Funktionieren des Wettbewerbsrechts auf den Märkten zuständig ist, die Mitgliedstaaten also keine widersprüchlichen Normen erlassen dürfen; Regelungen zur Grundstruktur des Binnenmarkts verbleiben jedoch noch immer in einer geteilten Zuständigkeit zwischen Gemeinschaft und Mitgliedstaaten. ${ }^{21}$ Im Bereich Energieversorgungssicherheit hat die Europäische Union in den derzeit gültigen Verträgen der Version von Nizza keine weitreichende Handlungsermächtigung. Unter diesem Gesichtspunkt stellen die Neuerungen im Vertrag von Lissabon einen tatsächlichen Richtungswandel dar. Für die Umweltpolitik besitzt die Union klassischerweise eine ausgreifende Kompetenz, auch über die Umweltverträglichkeits-Generalklausel aus Art. 6 EGV, die im Vertrag von Lissabon erhalten bleibt. ${ }^{22}$ Durch das neue Vertragswerk passt

21 Vgl. Beate Braams: Die Kompetenzordnung im Vertrag von Lissabon, in: Ingolf Pernice (Hrsg.): Der Vertrag von Lissabon: Reform der EU ohne Verfassung? Kolloquium zum 10. Geburtstag des WHI, Baden-Baden 2008, S. 115-134, hier S. 121-125.

22 Vgl. Art. 11 AEUV. 
sich die Europäische Union jedoch der vergleichsweise neuen Herausforderung der globalen Erwärmung an und weitet ihre Spielräume durch die Anerkennung der Notwendigkeit internationaler Vereinbarungen geringfügig aus.

\section{Schaubild 1: Neugestaltung des Primärrechts mit Auswirkungen auf die EU-Energie- und Klimapolitik im Vertrag von Lissabon}

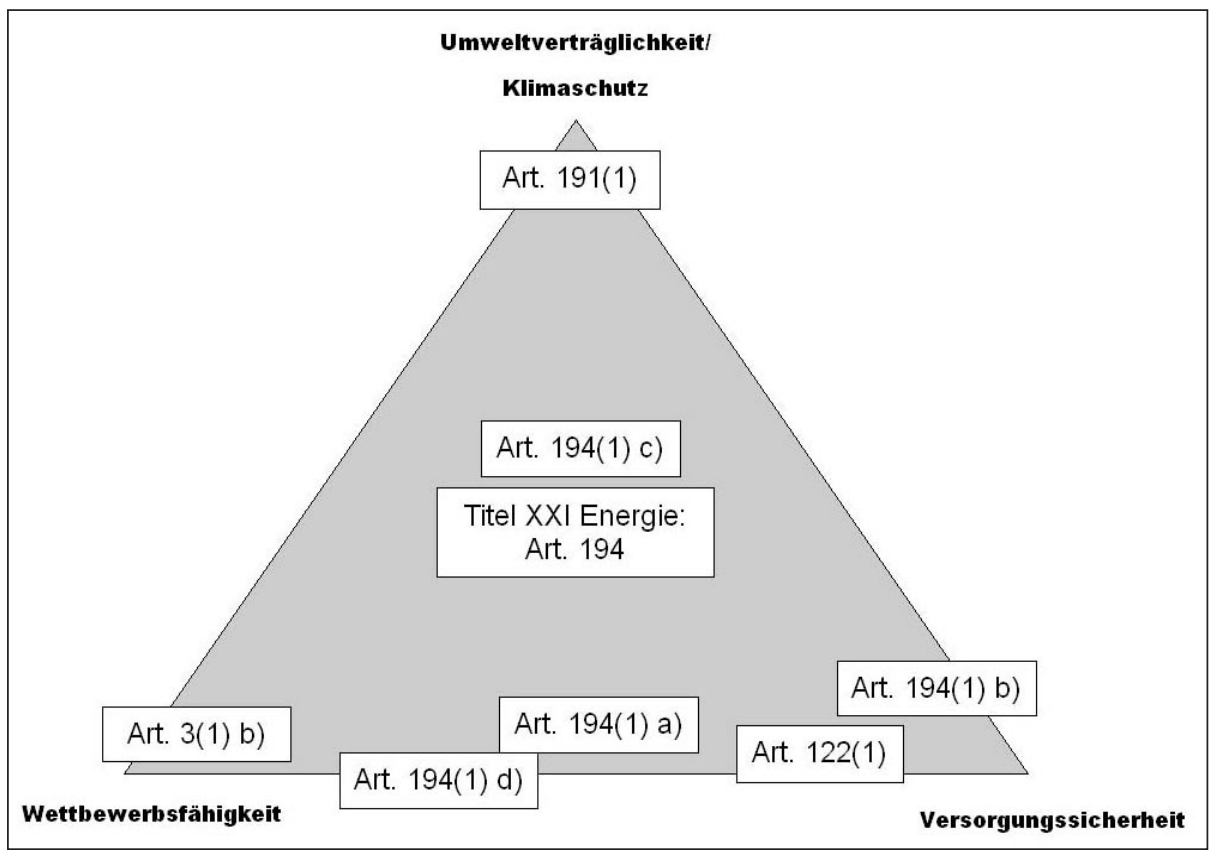

Quelle: Eigene Zusammenstellung der Artikel des AEUV.

Betrachtet man das Zieldreieck anhand seiner primärrechtlichen Verankerung im Vertrag von Lissabon (siehe Schaubild 1) und vergleicht dies mit der bisherigen Vertragsgrundlage von Nizza, so kann beobachtet werden, dass eine Ausbalancierung der drei strategischen Eckpunkte stattfindet. Die beiden bereits etablierten Zielsetzungen Wettbewerb und Umwelt wurden leicht ausgebaut, die Versorgungssicherheit hingegen erstmals mit einer eigenen Erwähnung ausgestattet und damit annähernd in den Rang der letzteren beiden aufgenommen.

\section{Die Vertragsänderungen als Legitimationsgrundlage für neue Projekte}

Die Europäische Union befindet sich seit März 2007, dem Zeitpunkt der Formulierung ihrer strategischen Beschlüsse für die Energie- und Klimapolitik und der Auflage des ersten Energieaktionsplans für die Jahre 2007-2009, auf dem schwierigen Weg der Umsetzung dieser Entscheidungen in Form konkreter Gesetzgebung. ${ }^{23}$ Dabei handelt es sich um drei Gesetzgebungspakete: Das ,Dritte Binnenmarktpaket` zur Weiterentwicklung des europäischen

23 Rat der Europäischen Union: Europäischer Rat, 8./9. März 2007. Der Energieaktionsplan befindet sich in Anlage I. Zur Umsetzung des Energieaktionsplans siehe unter anderem Institut für Europäische Politik (Hrsg.): EU Energy Policy Monitoring, abrufbar unter: http://energy.iep-berlin.de (letzter Zugriff: 25.11.2008). 
Marktes für Strom und Gas und zur Verschärfung des geltenden Wettbewerbsrechts ist seit September 2007 auf der Tagesordnung von Energieministerrat und Europäischem Parlament. Unter weit größerer öffentlicher Aufmerksamkeit wurde das im Januar 2008 vorgeschlagene Energie- und Klimapaket zunächst verhandelt und schließlich nach zähem Ringen bereits im Dezember 2008 durch den Europäischen Rat und das Europäische Parlament finalisiert. Mit dem Versorgungssicherheitspaket vom November 2008, das unter dem Titel „Zweite strategische Überprüfung der Energiepolitik“ firmiert, hat die Kommission schließlich alle drei Zielrichtungen der europäischen Energiestrategie vom März 2007 durch ein Legislativpaket im Rahmen der bestehenden primärrechtlichen Möglichkeiten bearbeitet. ${ }^{24}$

Sollte der Vertrag von Lissabon in naher Zukunft von allen Mitgliedstaaten ratifiziert werden, so könnte die Kommission auf Basis der darin enthaltenen Änderungen weitere Projekte mit gestärkter Legitimationsgrundlage angehen. Im Bereich der Wettbewerbsordnung dürften Regelungen von Seiten der Kommission mit deutlich mehr Durchschlagskraft angegangen werden. Durch die ,,ausschließliche Zuständigkeit“ bei der Festlegung von Wettbewerbsregeln ${ }^{25}$ in Kombination mit der Vorgabe, eine Politik zur Sicherstellung des Funktionierens des Energiemarktes ${ }^{26}$ zu betreiben, treffen diese Neuerungen den Kern des von der Kommission vorgelegten Pakets. Die darin enthaltenen Richtlinien- und Verordnungsentwürfe haben das Ziel, einen Rahmen zu schaffen, der ein diskriminierungsfreies und wettbewerbsorientiertes Funktionieren der Märkte für Strom und Erdgas gewährleisten soll. Dabei ist der Rückgriff auf Wettbewerbsrecht und Kartellrecht mit Blick auf die dominanten Energieversorgungsunternehmen einzelner Mitgliedstaaten als zweiter Schritt nach der Implementierung der Kommissionsvorschläge sehr wahrscheinlich. Auch wenn die Neuerungen des Vertrags von Lissabon hierfür eine erweiterte Handlungsgrundlage bieten, so darf nicht vergessen werden, dass die Europäische Union bereits heute in kaum einem anderen Bereich so erfolgreich und vielseitig aktiv ist, wie in der Politik zur Angleichung der Binnenmarktnormen und im Wettbewerbsrecht. Eine Erweiterung dieser Zuständigkeit und eine besondere Betonung des Energiesektors könnten an dieser Stelle nur noch marginale Ausweitungen der Eingriffstiefe von Seiten der Europäischen Kommission bedeuten. Schließlich hängen neue Marktregeln, ebenso wie die überwiegende Mehrheit aller Rechtsnormen, noch immer von der grundsätzlichen Zustimmung einer Mehrheit der Mitgliedstaaten im Rat ab, die durch eine Kompetenzausweitung allein keinesfalls gewährleistet ist. Die Durchsetzung konkreter wettbewerbsrechtlicher Verfahren gegen einzelne Mitgliedstaaten im Rahmen der Umsetzung von Richtlinien und Verordnungen oder kartellrechtliche Verfahren gegen einzelne Unternehmen könnten jedoch durch die explizite Zuständigkeit der Europäischen Union für die Sicherung des Marktes bei Verfahren vor dem Europäischen Gerichtshof von Bedeutung sein. Als weitaus bedeutsamer könnte sich jedoch die Vertragsänderung erweisen, durch die die „Interkonnektion der Energienetze“"27 als Handlungsfeld der Union festgelegt wird. Von der Öffentlichkeit beinahe unbemerkt, stellen Maßnahmen zum grenzüberschreitenden Infrastrukturausbau ein wichtiges Element des Energiebinnenmarktpakets dar. ${ }^{28}$ Dem Druck, diesen Ausbau eher fakultativ im Rahmen nicht verbindlicher Netzaus-

24 Siehe hierzu weiterführend Geden: Die Energie- und Klimapolitik der EU, 2008.

25 Art. 3 Abs. 1 Lit. b AEUV.

26 Art. 194 Abs. 1 Lit. a AEUV.

27 Art. 194 Abs. 1 Lit. d AEUV.

28 In diesem Zusammenhang erscheinen die Bestimmungen der Richtlinienvorschläge der Kommission für gemeinsame Vorschriften auf dem Erdgas- und Elektrizitätsbinnenmarkt als richtungsweisend. Vgl. Europäische Kommission: Vorschlag für eine Richtlinie des Europäischen Parlaments und des Rates zur Änderung der Richtlinie 2003/54/EG über die gemeinsamen Vorschriften für den Elektrizitätsbinnenmarkt, KOM (2007) 528, hier insbesondere Ziff. 8 bezüglich Art. 10 Abs. 2 und Ziff. 12 bezüglich Art. 22c); Europäische Kommission: 
baupläne für Energieversorger zu gestalten, der in dieser Form von Seiten einiger Mitgliedstaaten aufgebaut wurde, könnte mit einer konkreten primärrechtlichen Argumentationsgrundlage für eine Kompetenz der Union in diesem Bereich entgegengetreten werden.

Auf dem Gebiet der integrierten Umwelt- und Energiepolitik - und damit direkt verbunden: der Klimapolitik - kann die bisherige Handlungsermächtigung grundsätzlich als ausreichend bezeichnet werden. Die Richtlinien und Verordnungen des Energie- und Klimapakets stehen auf einer weitgehend soliden primärrechtlichen Grundlage, die die Union im Laufe der Jahre bis zur Vertragsrevision von Nizza erhalten hatte. ${ }^{29}$ Dennoch kann der Vertrag von Lissabon auch in diesem Bereich eine Ausweitung der Legitimationsgrundlage europäischen Handelns leisten. Die explizite Nennung von Energieeffizienz und Energieeinsparungen sind, ebenso wie die Entwicklung neuer und erneuerbarer Energiequellen, zentrale Gesichtspunkte des Legislativpakets vom Januar 2008. Sieht etwa die künftige Erneuerbare-Energien-Richtlinie ${ }^{30}$ ihre vertragliche Grundlage sowohl in Artikel 95 EGV zur Angleichung der Rechtsvorschriften im Binnenmarkt als auch in der Umsetzung einer umweltpolitischen Maßnahme, ${ }^{31}$ so würde nun eine einheitliche Rechtsgrundlage geschaffen, die rechtlichen Zweifeln an der Legitimation europäischer Politik über den Umweg geliehener Kompetenzen in diesem Bereich entgegentritt. Gleiches gilt für die zahlreichen, teils kleinteiligen Maßnahmen zur Steigerung der Energieeffizienz etwa bei Fahrzeugen, im Gebäudesektor oder bei Haushaltsprodukten. Eine Rückführung dieser Maßnahmen auf eine einzige Kompetenzgrundlage würde die Unsicherheit über die Rechtmäßigkeit und die richtige Gewichtung einer Kombination aus Binnenmarkt- und Umweltnormen vermindern. Schließlich könnte auch die Rolle des EU-Emissionshandelssystems, das in seiner Zielsetzung der Steigerung der Energieeffizienz, der Energieeinsparung und der dadurch zu erzielenden Emissionsminderungen dient, durch eine klare Handlungsermächtigung der Union in diesem Bereich weiter gestärkt werden.

Die wichtigste Legitimationsgrundlage für politisches Handeln, die der Vertrag von Lissabon in der Energiepolitik bietet, betrifft den Bereich Versorgungssicherheit. Hier mussten in der Vergangenheit die größten Defizite konstatiert werden. Diese resultierten einerseits aus der nationalen Dominanz in dieser äußerst sensiblen Dimension der Energiepolitik, zum anderen aus der defizitären Kompetenzgrundlage für gemeinsame Rechtsetzungsverfahren. Durch die explizite Nennung der Versorgungssicherheit als Ziel europäischer Energiepolitik und durch die Einbeziehung dieses Faktors in die Entwicklung von Maßnahmen im Falle gravierender Schwierigkeiten bei der Versorgung mit Gütern, insbesondere im Energiebereich, ${ }^{32}$ wurde eine Rechtsgrundlage geschaffen, die in Zukunft für proaktive Schritte der Union genutzt werden kann. Denkbar sind diesbezüglich Richtlinien zur Speicherung von Erdgas oder die Entwicklung eines rechtlich ausgestalteten präventiven Krisenreaktionsmechanismus für die Solidaritätsklausel aus Art. 122 AEUV. Neben Nachhaltigkeit und Wettbewerbsfähigkeit stellt die Versorgungssicherheit den einzigen Bereich europäischer Energiepolitik dar, in dem die Kommission bislang vor der Anwendung konkreter Legisla-

Vorschlag für eine Richtlinie des Europäischen Parlaments und des Rates zur Änderung der Richtlinie 2003/ 55/EG über gemeinsame Vorschriften für den Erdgasbinnenmarkt, KOM (2007) 529, hier insbesondere Ziff. 8 bezüglich Art. 9 Abs. 2 und Ziff. 14 bezüglich Art. 24c).

29 Titel XIX, Art. 174-176 EGV.

30 Europäische Kommission: Vorschlag für eine Richtlinie des Europäischen Parlaments und des Rates zur Förderung der Nutzung von Energie aus erneuerbaren Quellen, KOM (2008) 19. Die rechtsverbindliche Version der Richtlinie lag bei Redaktionsschluss noch nicht vor.

31 Als erste Rechtsgrundlage wird in der noch nicht veröffentlichten Fassung der Richtlinie zur Förderung erneuerbarer Energien Art. 175 Abs. 1 EGV genannt, für Art. 15-17 der Richtlinie gilt Art. 95 EGV.

32 Art. 122 AEUV. 
tivverfahren zurückschreckte. Dies liegt in erster Linie darin begründet, dass eine ,Kompetenzanleihe" aus einem anderen Politikfeld nur schwer zu bewerkstelligen war. Erst mit der Schaffung einer expliziten Kompetenz für die Energieversorgungssicherheit im Vertrag von Lissabon würde sich dies grundsätzlich ändern.

\section{Die psychologische Wirkung der Vertragsänderungen für die Europäische Union als Akteur in der Energie- und Klimapolitik}

Neben den primärrechtlichen und legitimationsbildenden Aspekten der Vertragsänderungen kann die Gestaltung neuer Kompetenzrahmen durch das Inkrafttreten des Vertrags von Lissabon die Eigenwahrnehmung der Europäischen Union als Akteur in ihrer internen und externen Dimension grundsätzlich verändern.

Im Rückblick auf die bisherige Entwicklung europäischer Energiepolitik fällt auf, dass ihr Erfolge bislang nicht primär durch den Mangel an ausreichenden Kompetenzen verwehrt blieben, sondern dass ein zweiter gewichtiger Hemmfaktor für die Begrenztheit europäischer Strategien verantwortlich zu sein scheint: Trotz grundsätzlicher Übereinstimmung in den groben Ausrichtungen fielen Mitgliedstaaten stets in die Kategorie nationaler Interessen zurück, sobald strategische Beschlüsse in konkrete Maßnahmen umgesetzt werden sollten. ${ }^{33}$ Dies ist in den allermeisten Fällen auf die grundsätzliche Problematik zurückzuführen, dass die Mitgliedstaaten und ihre Regierungen die dominanten Akteure im Rahmen der Energiepolitik darstellen. In besonderem Maße traf diese Beobachtung in der Vergangenheit auf Ereignisse zu, in denen bilaterale Deals oder Abmachungen mit Drittstaaten einen vermeintlichen Vorteil für den jeweiligen Mitgliedstaat oder ein ansässiges Energieunternehmen bedeuten könnten. ${ }^{34}$ Die Tatsache, dass die Europäische Union mit der endgültigen Ratifikation des Vertrags von Lissabon eine explizite energiepolitische Kompetenz erhalten wird, könnte sie als ernst zu nehmender Akteur auf dem Gebiet der Energiepolitik ins tagespolitische Rampenlicht rücken lassen. Selbst wenn sich in der praktischen Ausrichtung keine fundamentalen Richtungsänderungen infolge der Vertragsänderungen ereignen dürften, so könnte sich die Selbstwahrnehmung der Europäischen Union als eigenständiger rechtsetzender Akteur weiter fortentwickeln und zu einer größeren Selbstverständlichkeit europäischer Handlung im Rahmen der Energiepolitik führen. ${ }^{35}$ Dies würde auch integrationsskeptische Mitgliedstaaten von der Handlungsfähigkeit und einem möglichen Mehrwert europäischer Maßnahmen in diesem Bereich überzeugen. Schließlich dürfte potenziell auch ein entsprechendes Grundsatzurteil des Europäischen Gerichtshofs, argumentativ auf den Bestimmungen des Energietitels basierend, einen ähnlichen Effekt erzielen. ${ }^{36}$ Der subjektive Wahrneh-

33 Vgl. Florian Baumann/Jürgen Turek: Die europäische Energiepolitik im Vertrag von Lissabon, in: Werner Weidenfeld (Hrsg.): Lissabon in der Analyse. Der Reformvertrag der Europäischen Union, Baden-Baden 2008, S. 157-169, hier S. 163.

34 Einige Beispiele der jüngeren Vergangenheit betreffen die bilateralen Abmachungen zum Bau der Nord Stream-Pipeline zwischen Deutschland und Russland oder die Debatte über Beteiligungen an der NabuccoPipeline, die Erdgas aus dem kaspischen Raum nach Europa leiten soll. Zu letzterem Beispiel siehe unter anderem Severin Fischer: Verrat an Europa? Ungarns pragmatische Energieaußenpolitik im Spannungsfeld zwischen Diversifizierung und Versorgungssicherheit, SWP-Diskussionspapier der FG 1 19/2007.

35 Vgl. Kuhlmann: Kompetenzrechtliche Neuerungen im europäischen Energierecht nach Lissabon, 2008, S. 26.

36 Bereits in der Vergangenheit prägten Urteile des Europäischen Gerichtshofs die weitere Entwicklung der Energiepolitik auf europäischer Ebene. Als Beispiele können folgende Urteile angeführt werden: Europäischer Gerichtshof: Rs. 6/64 (Costa/E.N.E.L.), Slg. 1964, 1253; Europäischer Gerichtshof: Rs. C-320/91 (Corbeau), Slg. 1993, I-2533; Europäischer Gerichtshof: Rs. C-393/92 (Gemeente Almelo u.a./Energiebedrijf Ijsselmij), Slg. 1994, I-1477; Europäischer Gerichtshof: Rs. C-379/98 (PreussenElektra), Slg. 2001, I-2099. Siehe ausführlicher unter anderem Lisa Conant: Justice Contained. Law and Politics in the European Union, Ithaca/London 2002; Geden/Fischer: Die Energie- und Klimapolitik der Europäischen Union, 2008, S. 55-59. 
mungswandel, der die Europäische Union von einem energiepolitischen Lückenfüller zu einem dominanten Akteur in diesem Politikfeld aufsteigen lässt, stellt einen langwierigen Prozess dar, der jedoch durch eine kompetenzrechtliche Kodifizierung seinen Ausgangspunkt nehmen könnte.

Eine noch bedeutendere psychologische Funktion dürfte der Energietitel in den Außenbeziehungen der Europäischen Union einnehmen. Wichtige Akteure der internationalen Energiepolitik, so etwa Russland und die OPEC-Staaten, nehmen Europa bei Weitem nicht als den zentralen Spieler auf dem globalen Energiemarkt wahr. Vielmehr sind es einzelne Unternehmen und die Mitgliedstaaten der Europäischen Union, die als entsprechendes Gegenüber aufgefasst werden. ${ }^{37}$ Diese Wahrnehmung resultiert weniger aus der kompetenzrechtlichen Verfassung der Europäischen Union, als vielmehr aus dem Interesse der großen Exporteure fossiler Rohstoffe, die sich durch die Konzentration auf einzelne kleinere Akteure und das Stützen auf entsprechende nationale Ressentiments ${ }^{38}$ eine bessere Position in Verhandlungen über Öl- und Gaslieferverträge versprechen. Durch die Entwicklung einer energiepolitischen Kompetenz allein wird dieser Zustand zwar kaum verändert, doch könnten die europäischen Institutionen, allen voran die Kommission, die Chance ergreifen, ihre aufgewertete Rolle in der internationalen Energiepolitik zu nutzen und Europa als einheitlichen Akteur auftreten zu lassen. Die Fortsetzung einer Politik zur Vollendung des Energiebinnenmarktes wird hierfür ohnehin eine Voraussetzung schaffen, welche die nationalen Möglichkeiten der Einflussnahme verringert. Durch die Vertragsergänzungen wird der Prozess der Entwicklung eines kohärenten Akteurs ,Europa‘ in den internationalen Energiebeziehungen somit noch weiter bestärkt.

Schließlich könnten die Anerkennung der Problematik des Klimawandels im Vertragswerk sowie die primärrechtlich verankerte Selbstverpflichtung Europas, sich für ein globales Klimaschutzabkommen einzusetzen, psychologisch den größten Mehrwert bringen. Die Europäische Union beansprucht für sich eine Führungsrolle in der internationalen Klimapolitik. Um diese auch praktisch wahrzunehmen, bietet die globale Klimakonferenz im Dezember 2009 in Kopenhagen einen geeigneten Anlass. Dass es innerhalb einer Gemeinschaft von 27 Mitgliedstaaten mit spezifischen Interessen sowie Industrie- und Energiestrukturen möglich ist, eine solidarische Lösung für die Klimaschutzbemühungen aller zu finden, haben die Gipfelergebnisse des Europäischen Rates vom 11./12. Dezember 2008 gezeigt. ${ }^{39}$ Auch wenn nicht alle Beteiligten zufrieden mit dem Ergebnis waren, so dürfte dieses dennoch ein positives Signal für die internationalen Verhandlungen aussenden. Die Kodifizierung der Pflicht zum Klimaschutz ist dabei nur ein kleiner Baustein, könnte der Weltgemeinschaft jedoch gleichermaßen demonstrieren, dass Europa seine Verpflichtung ernst nimmt und diese in seine, Verfassung" integriert.

\section{Zwei Schritte vor, ein Schritt zurück: Die nationale Dominanz in der Energiepolitik bleibt als limitierende Komponente erhalten}

Trotz der durchaus positiven Bewertung der energiepolitischen Entwicklungen, die sich infolge einer Ratifikation des Vertrags von Lissabon vollziehen könnten, bietet das revidierte Primärrecht keineswegs einen eindeutigen Fingerzeig hin zu einer ausschließlichen

37 Vgl. Severin Fischer: Energieversorgungssicherheit: Was kann Europa leisten?, in: Gesellschaft-WirtschaftPolitik 4/2008, S. 407-418.

38 Beispielsweise das historisch belastete deutsch-polnische Verhältnis.

39 Vgl. Rat der Europäischen Union: Europäischer Rat (Brüssel), 11./12. Dezember 2008. Schlussfolgerungen des Vorsitzes, Dok. 17271/08. 
Zuständigkeit der Europäischen Union in diesem Politikfeld. Vielmehr fällt auf, dass die Mitgliedstaaten während der Verhandlungen über den Vertrag von Lissabon großen Wert auf die Souveränität staatlicher Entscheidungen über die Nutzung der Ressourcen und die Wahl ihres Energiemixes legten. Nicht umsonst taucht diese Bestimmung nicht nur im neu geschaffenen Energietitel auf, ${ }^{40}$ sondern wiederholt sich auch im Umwelttitel. ${ }^{41}$ Eine solche doppelte Absicherung macht deutlich, dass die Regierungen keineswegs gewillt waren, Entscheidungen über die grundlegendsten Elemente der Energiepolitik an eine supranationale Ebene abzutreten.

Die Verfechter dieser Einschränkung europapolitischer Kompetenz im Energiebereich lassen sich leicht vereinfacht in zwei Staatengruppen aufteilen: Zunächst treten rohstoffreiche Mitgliedstaaten für eine freie Entscheidung über die Nutzung ihrer natürlichen Ressourcen ein. Hierzu gehören in erster Linie Großbritannien, die Niederlande und Dänemark. Für sie wäre ein europäischer Eingriff und eine befürchtete europäische Regulierung der Nutzung ihrer Bodenschätze ein tiefgreifender Einschnitt in ihre staatliche Souveränität, der gegenüber der eigenen Bevölkerung nur schwer zu vertreten wäre. Eine zweite Gruppe von Staaten, darunter Deutschland und Österreich, zeichnen sich im europäischen Vergleich durch eine eher exklusive Stellung aus: Sie lehnen die Nutzung der Atomkraft ab. Aufgrund historischer Erfahrungen und gesellschaftlicher Prozesse hat sich hier ein eher skeptischer Blick auf die Nutzung der Kernenergie herausgebildet. Berücksichtigt man, dass die Europäische Union nicht nur aufgrund des noch immer gültigen EURATOM-Vertrages, sondern auch wegen der grundsätzlich positiven Haltung gegenüber der Atomkraft in Europäischem Parlament und Kommission schon immer ein kerntechnologiefreundliches Portfolio besaß, kann die Skepsis gegenüber einer Europäisierung der Entscheidung über den Energiemix in diesen Staaten durchaus nachvollzogen werden. Für beide Staatengruppen ist die nationale Kontrolle über Ressourcen und Energieträger ein entscheidender Faktor ihrer staatlichen Souveränität.

Schließlich findet sich in Erklärung Nr. 35 zum Vertrag von Lissabon eine weitere integrationsbeschränkende Vereinbarung, welche die Grundeinstellung europäischer Regierungen zur ,Einmischung Europas` in nationale Energiepolitiken widerspiegelt. In dieser Erklärung, die dem Vertrag von Lissabon als Anhang beigefügt wurde, wird deutlich hervorgehoben, dass das Recht der Mitgliedstaaten zum Erlass nationaler Regelungen zur Sicherung der Energieversorgung unberührt bleibt. Dies schränkt die in Art. 194 AEUV verfügte europäische Politik zur Gewährleistung von Energieversorgungssicherheit im Krisenfall deutlich ein, denn Mitgliedstaaten können im Zweifel auf Grundlage des dann gültigen Vertrags Maßnahmen ergreifen, die nicht notwendigerweise dem Wohl der gesamten Gemeinschaft zuträglich sind.

So bleibt zu fragen, welchen Effekt die angeführten limitierenden Faktoren für eine europäische Energiepolitik hervorrufen könnten. Ist ihr Einfluss auf die konkrete Gestaltung europäischer Gesetzgebung tatsächlich bedeutend?

Die Kontrolle der Mitgliedstaaten über ihre nationalen Ressourcen und die Entscheidung über den Energiemix bergen eher eine potenzielle, als eine konkrete Gefahr für die Entwicklung einer europäischen Energiepolitik. Nichtsdestotrotz können Fälle konstruiert werden, in denen sich Mitgliedstaaten auf ihre vertraglich fixierten Rechte berufen und damit europäische Strategien aushebeln könnten. Ein Beispiel hierfür stellt die Entwicklung und Förderung erneuerbarer Energien dar. In ihrem Richtlinienentwurf vom Januar 2008 schlug die

40 Art. 194 Abs. 2 AEUV.

41 Art. 192 Abs. 2 Lit. c AEUV. 
Europäische Kommission erstmals einen Gesetzestext vor, der für jeden Mitgliedstaat differenzierte Ziele für den Anteil erneuerbarer Energien im Jahr 2020 rechtsverbindlich festschreiben soll. ${ }^{42}$ In der Entscheidung des federführenden Industrieausschusses des Europäischen Parlaments ging der zuständige Berichterstatter Claude Turmes sogar noch weiter und forderte rechtsverbindliche Zwischenziele für die Zeit bis zum Jahr 2020. ${ }^{43}$ Während sich im Umweltministerrat die Kritik auf andere Aspekte des Vorschlags konzentriert hatte, ${ }^{44}$ könnte sich dies einige Jahre nach Inkrafttreten der Richtlinie grundlegend ändern. Sollte ein Mitgliedstaat seinen nationalen Zielwert absehbar verfehlen, so läge eine Berufung auf die Entscheidungshoheit über den Energiemix und den ungerechtfertigten Eingriff der Europäischen Union in diesem Bereich durch die Festsetzung eines verbindlichen Anteils erneuerbarer Energien durchaus im Bereich des Möglichen. Dieses Argument würde in jedem Fall zur Abwehr eines Vertragsverletzungsverfahrens genutzt. Auch ein gesellschaftlicher Stimmungswandel, wie er zuletzt im Verhältnis zur Zielfestlegung für den Anteil erneuerbarer Energien im Verkehrssektor zu beobachten ist, zeigt deutlich, dass die Mitgliedstaaten im Zweifelsfall schnell dazu neigen, gemeinsame Beschlüsse im Zuge sich wandelnder Rahmenbedingungen für obsolet zu erklären. Ist dies auf Grundlage eines Vertragsartikels möglich, könnte das Gesamtprojekt, Erneuerbare Energien ' auf Grundlage temporärer Ereignisse oder gesellschaftlicher Stimmungsveränderungen auf europäischer Ebene gefährdet werden.

Ein ähnlicher Fall ließe sich für die Bestimmungen des EU-Emissionshandelssystems konstruieren. Im Vorfeld der entscheidenden Verhandlungen des Europäischen Rates im Dezember 2008 erhob eine Koalition mittelosteuropäischer Staaten unter Führung Polens und im Einklang mit der italienischen Regierung grundsätzliche Bedenken gegen die von der Kommission vorgeschlagenen Bestimmungen für die dritte Handelsperiode dieses Vorzeigeprojekts europäischen Klimaschutzes. ${ }^{45}$ Im Kontext zukünftiger Verhandlungen oder einer Überprüfung der Richtlinie zu einem späteren Zeitpunkt, erscheint es durchaus denkbar, dass Polen oder ein anderer Staat den aus den nun gültigen Bestimmungen resultierenden Effekt der Abkehr von der emissionslastigen Steinkohle als Eingriff in die nationale Souveränität im Zusammenhang mit der Entscheidung über die Nutzung einzelner Energieressourcen anführt, insbesondere mit Blick auf die einschränkenden Vorgaben im Umwelttitel des Vertrags von Lissabon. Im Sinne nationaler Maßnahmen zur Gewährleistung der Energieversorgungssicherheit wäre es auch durchaus denkbar, dass die Gefahr, die eine Kompensation des hohen Kohleanteils in der polnischen Primärenergieerzeugung durch emissionsarmes russisches Erdgas mit sich brächte, als Einschränkung nationaler Sicherheitsbedürfnisse in diesem Bereich verstanden wird.

Durch diese Beispiele wird deutlich, dass die limitierenden Faktoren einer zukünftigen primärrechtlichen Verankerung europäischer Energiepolitik in den meisten Fällen etwa de-

42 Europäische Kommission: Vorschlag für eine Richtlinie zur Förderung der Nutzung von Energie aus erneuerbaren Quellen, 2008, insbesondere Art. 1, Art. 3 und Anhang I Teil A.

43 Europäisches Parlament: Bericht über den Vorschlag für eine Richtlinie des Europäischen Parlaments und des Rates zur Förderung der Nutzung von Energie aus erneuerbaren Quellen (KOM(2008)0019 - C6-0046/2008 2008/0016(COD)), Bericht des Ausschusses für Industrie, Forschung und Energie, Berichterstatter Claude Turmes, A6-0369/2008 vom 26.9.2008. Siehe dazu unter anderem Institut für Europäische Politik (Hrsg.): EU Energy Policy Monitoring, abrufbar unter: http://energy.iep-berlin.de/php/4_erneuerbar_entwicklung18.php (letzter Zugriff: 25.11.2008).

44 Kritik äußerten die Mitgliedstaaten vor allem an der geplanten Einführung eines europaweiten Handels mit Herkunftszertifikaten für erneuerbare Energien.

45 Vgl. unter anderem Jennifer Rankin: Little movements on EU's climate plans, in: European Voice, 21.10.2008; Cerstin Gammelin: Stillstand beim Klimaschutz, in: Süddeutsche Zeitung, 21.10.2008. 
ckungsgleich mit den Zielkonflikten zwischen Versorgungssicherheit, Wettbewerbsfähigkeit und Umweltschutz verlaufen. Solange einer der drei Bereiche noch immer vornehmlich unter nationaler Kontrolle bleibt, wird sich die potenzielle Gefahr des Untergrabens europäischer Strategien durch nationale Alleingänge nicht verhindern lassen. Während dies in der konkreten Ausgestaltung der Gesetzgebung derzeit nur selten von Bedeutung ist, zeigen das Aufkommen der Finanzkrise oder vergleichbarer Erscheinungen, dass sich ein gemeinsamer Nenner, insbesondere in Fragen des Klimaschutzes, noch nicht verfestigt hat. In diesen Momenten könnte sich die Beschränktheit europäischer Energie- und Klimapolitik als prekär erweisen.

\section{Europäische Energie- und Klimapolitik auch ohne den Vertrag von Lissabon?}

Während in den Institutionen der Europäischen Union ein weitgehendes Denkverbot über Alternativpläne zum Vertrag von Lissabon herrscht, werden in den Hauptstädten Europas durchaus Zukunftsszenarien diskutiert, die von einem Scheitern des Reformvertragprojekts ausgehen. Was würde ein solches Scheitern für den Bereich der Energie- und Klimapolitik bedeuten?

Da der Vertrag von Lissabon keine „Systemrevolution“ darstellt, sondern eher eine Modernisierung und Positivierung bestehenden Rechts vornimmt, ${ }^{46}$ fallen auch die Folgen seines Nicht-Inkrafttretens für die Bearbeitung spezifischer Politikfelder nicht so schwer ins Gewicht wie es für eine ausbleibende Reform der Institutionen der Fall wäre. Dennoch gilt die Energie- und Klimapolitik, wie hier gezeigt, zu Recht als einer der Gewinner des Lissabonner Vertrags und würde durch die Begrenzung der Kompetenzen auf das Niveau von Nizza wohl großer Bereiche zukünftiger Handlungsfähigkeit beraubt. Schließlich darf dabei jedoch nicht vergessen werden, dass sowohl die Beschlüsse des Europäischen Rates vom März 2007 als auch die ambitionierten Pläne der Europäischen Kommission hinsichtlich einer europäischen Energiepolitik auf der Grundlage des Primärrechts von Nizza verfasst sind und damit in ihrer ursprünglichen Konzeption nicht von einer weiter europäisierten Energiepolitik ausgehen durften. Gleiches gilt für die lancierten Gesetzgebungspakete zum Elektrizitäts- und Erdgasbinnenmarkt, zur Klimapolitik und zur Versorgungssicherheit. Alle Rechtsgrundlagen wurden auf Basis des gültigen Vertrags von Nizza konzipiert und sollten somit auch auf dieser Grundlage verabschiedet werden.

Die scheinbare Funktionsfähigkeit europäischer Energiepolitik auf der Basis von Nizza soll jedoch keinesfalls dazu verleiten, eine explizite Rechtsgrundlage für unnötig zu erachten. Letztere brächte europäischen Handlungen einen deutlichen Zuwachs an Legitimation, wäre für die Wahrnehmung und Darstellung der Europäischen Union in ihrer inneren und äußeren Dimension von entscheidender Bedeutung und könnte schließlich in aufkommenden Krisenmomenten ein deutlich höheres Maß an Rechtssicherheit für europäische Projekte mit sich bringen. Nichtsdestotrotz löst auch der Vertrag von Lissabon noch nicht die bestehenden Zielkonflikte europäischer Energiepolitik. Eine Garantie, dass Wettbewerbsfähigkeit, Versorgungssicherheit und Umweltverträglichkeit in der Energiepolitik in Einklang zu bringen und auf europäischer Ebene erfolgreich zu behandeln sind, bietet auch dieses Reformwerk nicht. Hierfür bedürfte es einer grundsätzlichen Strategiedebatte sowie eines weiteren Reformprojekts, das derzeit noch nicht absehbar ist.

46 Vgl. Peter-Christian Müller-Graff: Der Vertrag von Lissabon auf der Systemspur des Europäischen Primärrechts, in: integration 2/2008, S. 123-144. 